\title{
Imensko kazalo
}

\section{A}

Abramić, Mihovil 37, 38, 51

Adam Parižanin 387, 388

Adler, Alfred 430

Adler, Viktor 440

Afrić, Vjekoslav 20, 464, 465, 468, 470, 475, 480

Alberti, Leon Battista 391

Albini, Srećko 472, 474

Aleksandar Makedonski 29

Aleksandrovna, Irina 466

Alkej, pjesnik 299

Alkmeon iz Krotona 167

Aljinović, Grozdana 423

Amenofis (Amenhotep) III., faraon 43

Anakreont 34, 299

Anastazije, mučenik 387-388

Anderson, Olive 436, 446

Andreas-Salome, Lou 434

Andrić, Ivo 69, 98, 305, 311, 313

Andrić, Nikola 438

Angeli Bertinelli, Maria Gabriella 370, 378

Anić, Šime 38,51

Anić, Tomislav 427, 446

Anić, Vladimir 38, 51

Antić, Miroslav (Антић, Мирослав) 10, 15, 315-326

Antonino Pio, car 373-374

Anušić, Nikola 20, 453, 454, 459, 461

Aposotolova Maršalevski, Magdalena 409, 419

Arenas, Reinald 344

Arhiloh, pjesnik 25, 299

Ariès, Philippe (Arijes, Filip) 21-22, 172, $177,422,446$

Aristotel 13, 33, 34, 51, 106, 329, 334

Artić, Miroslav 15

Artuković, Andrija 474

Asino, Rosalba 442, 446

Astuti, Rita 316, 325

Atebod, Tito Flavije 369-370

Aterig (Aterix) 369-370

August, car 40,382
Augustin Aurelije 341

Auzonije, pjesnik 377

\section{B}

Babić, Ivo 389, 395

Babić, Ljubo 308

Baccarini, Elvo 243, 246

Bagić, Krešimir 192, 196, 200

Bahr, Andreas 433, 446

Bahtin, Mihail 13, 60, 67, 147, 150-151, 152, 153, 154, 156

Bailey,Tara 484, 492

Bajčeta, Vladan 9, 12, 79, 87, 381, 396

Banović, Snježana 20, 468, 470, 474, 480

Banti, Alberto Mario 485, 492

Banjanin, Ljiljana 169, 177

Barac, Antun 465, 480

Barać, Stanislava 13, 145

Baratin, Luka, nadbiskup 392

Barbarič, Štefan 12, 93, 94-95, 101, 102

Barbarić, Damir 330, 338

Baričević, Mato 28

Barlle, Petar 405

Basara, Svetislav 344

Basić, Ivan 384, 395

Baskin, Andrea 352, 354, 355

Bass Verzár, Monika 367, 378

Batušić, Nikola 446

Baudelaire, Charles 432, 445

Baudrillard, Jean (Bodrijar, Žan) 306, 313

Baumann, Ursula 433, 446

Bavčević, Branko 54

Becić, Vladimir 431

Beck (Bek), Vladimir 464

Beč, Dušan, vatrogasac 466

Bedford, James 242

Begović, Božena 463, 466, 481

Beiser, Frederick Charles 329, 337

Beit-Hallahmi, Benjamin 316, 325

Bela IV., kralj 389

Benn, Gottfried (Ben, Gotfrid) 13, 117 
Benjamin, Walter (Бенјамин, Вамтер) 13, $14,130-131,135-136,139,142-143,144$, $145,162,164,445,446$

Berdjajev, Nikolaj 443

Berg, Alban 435

Berger, Peter L. $116,121,125$

Berkeš, Đuka 464, 480

Bernhard, Thomas 117

Berry, Peter 262, 278

Bervaldi, Josip 383, 384, 395

Bešević, Ivanka (БЕшевић, Иванка) 23, 51, 104

Binički, Aleksandar 466, 468

Binički, S. 478

Biondić, Marin 14, 220, 222

Biraben, Jean-Noel 453, 461

Birk, Stine 366, 367, 368, 376, 377

Biti, Vladimir 59, 67, 131, 135, 143, 145, 192, 200

Bitroff, Paulus 371

Blackshaw, Gemma 439, 446

Blanchot, Maurice (Blanšo, Moris) 14, 159, 163, 164, 215, 221, 222

Blažeković, Zdenko 470, 475

Bloom, Harold 13, 153, 156

Bogdani, Vitaliano 37

Bogdanović, Danijel 354,358-359

Bogdanović, Milan 305, 313

Boić, Gemma 437

Bolf, Ivan (Bollff, Joannes /Bollff /Bolff, Boliuff/, Januss), gradski prisjednik 40, 406, 409, 412, 414

Boltzmann, Ludwig 435

Bonfante, Larisa 367, 378

Borges, Jorge Luis 344

Borko, Božidar 90

Borovečki, Ana 453, 461

Borstelmann, Thomas 262, 278

Bošnjak, Branko 51, 184, 189, 214, 223

Bowersock, Glen Warren 372, 378

Bradley, Ben 327, 337, 382, 397

Brajković, Tomo 285

Brajović, Tihomir (Брајовић, Тихомир) 72, 87

Brandt, Olof 383, 397

Brecht (Breht), Bertolt 106

Brent, Sandor B. 316, 326

Brkić, Branislav 53
Brkić, Dušan 491

Brnčić, Jadranka 22, 83, 87, 104, 114

Brown, Peter 372, 378

Brown, Ron M. 436, 437, 446

Brozović, Dalibor 490, 491

Brozović, Domagoj 262, 263, 275, 278

Brunelli, Vitaliano 37, 52

Bruner, Jerome 124, 125

Brut, Marko Junije, senator 27

Bruun, Christier 275, 376, 377, 383, 296, 397

Budak, Mile 464, 465, 472, 477, 478

Budak, Pero 486, 490

Budžejr bin Zuhayr 297

Bui, Boris 22

Bujas, Ramir 333

Bukarica-Ivanović, Miljenko 298

Bulić, Frane, don 383, 384, 385, 395, 438

Buljac, Miljenko 211, 222

Buntak, Franjo 402, 419

Burgess, Anthony 16, 237

Butler, Shane 377

Buzolić, Stjepan 285, 288

\section{C}

Caban, Petar 383, 395

Cambi, Nenad 38, 52, 369, 378

Campbell Thompson, Reginald 263, 278

Camus, Albert 173, 178

Cankar, Ivan 98

Cannadine, David 427, 436, 439, 447

Car, Marko 27, 28, 29, 52, 56, 285

Carducci, Giosuè 282

Carnegie, Dale 30

Carroll, Maureen 365, 366, 367, 370, 377

Cassirer, Ernst 329

Castiglia, Gabriele 383, 397

Cavalcanti, Guido 282, 291

Cazes, Bernard 255, 259

Cellini, Benvenuto 45, 52

Cezar Julije, car 26, 27, 29, 34

Cezarnije Avito (Caesernius Avitus) 373, 374

Chamoux, François 382, 396

Cheng'en, Wu 263, 278

Chevalier, Jean 353, 363 
Chioffi, Laura 383, 396

Choron, Jacques 263, 278

Churchland, Paul M. 119, 120, 125

Ciceron, Marko Tulije 367

Cipiko, Koriolan 392, 396

Claccich, kanonik 37 v. Klačić

Claesges, Ulrich 329, 337

Cohen, Hermann 329

Cohn, Dorrit 118, 125

Comini, Alessandra 439, 447

Cooley, Alison E. 382, 396

Corbusier, Le, Charles-Édouard (Jeanneret) 430

Corcoran, Simon 376, 377

Crepuljarević, Vesna D. 207, 210

Crespo Fernandéz, Eliecer 195, 200

Crljen, Danijel 464,480

Crnković, Milan 316, 325

Crnobori, Marija 468, 470, 475, 480

Crnjanski, Miloš 69, 304, 305, 313

Croce (Kroče), Benedetto 10, 93, 282

Curl, James 436, 447

Curtis, Antonio de (Totò) 487

Cuscito, Giuseppe 367,378

Cvetušić, Mihalj 404

Cvijić, Jovan 229, 235

Cvijović Javorina, Ivana $70,72,73,78,83$, $87,104,114,130,135,145,148.152$, $156,211,222,223,230,231,232,235$, 468, 480

Cvitan, Dalibor 491

Czigany, Dezso 433

Czoczelly, Susana 412

\section{Č}

Čačić, Petar (Џаџић, Петар) 69,87

Čale Frano 52, 281, 285, 290, 291, 292

Čeh Stergar, Jožica 261

Čehok, Ivan 328, 334, 338, 337

Čehov, Anton Pavlovič 13

Čengić, Enes 466, 468, 480

Čokrljan, Jovan 353

Čorak, Željka 251
Ć

Ćepulić, Vladimir 461

Ćirilov, Jovan 105

Ćorović, Vladimir 385, 396

\section{D}

Dabo, Radoslav 90

Damaz, papa 382, 383

Damjanov, Sava 353, 363

Daničić, Đuro 484, 493

Daniele, Pino 487

Dante, Alighieri 10, 23, 24, 34, 36, 45, 47, $52,56,155,285$

Darić, Juraj (Darich, Georgius) 410

Darwin, Mike 242, 247

Davis, Stephen T. 18, 341-344, 349

Delany (Delejni), Samuel R. 118

Deleuze, Gilles 163, 176, 177

Delić, Jovan (Аемић, Јован) 81, 87

Delonga, Vedrana 384, 385, 396

Demeter, Dimitrije 478

Demichel, Dino 41

Demosten 26, 27

Derrida, Jacques 163, 274-275

Desnica, Boško 17, 282, 283, 285, 288, 289, 291

Desnica, Ksenija 491

Desnica, Vladan 19, 12, 13, 14, 15, 17, 18, $20,21,22,23-51,52,53,54,55,56,57-$ $66,67,68,69-86,88,89-100,101,102$, 103-114, 115, 117-125, 126-127, 129-144, $145,146,147-156,157,159-164,165-177$, $178,179-189,190,191-200,201,203-$ 209, 210, 211-222, 223, 224, 225-235, 236, 237-246, 247, 249-258, 259, 261277, 278, 279, 280, 281-291, 292, 381, 395, 483-492, 493

Desnica, Uroš 22, 27, 52, 283, 490, 491

Desnica, Uroš (otac Vladanov) 17

Deutsch, Albert 28

Dević, Đ. 464

Dežman, Milivoj 434, 442

Diehl, Ernst 382, 396

Dileja, Mile 17, 317

Dimitrijev, Fjodor 466

Divković, Mirko 39, 52 
Dobre, dakon $385,387,388$

Dobronić, Lelja 392, 396, 418, 419

Dojkić, Andrej 113

Dokaza, Ivan 354, 360-361

Dominik Michael, dužd 387

Domović, Želimir 38,51

Donadini, Ulderiko 441, 447

Donat, biskup 384

Donat, Branimir 44, 52

Donati, Angela 370, 378, 441, 447

Donati, Ivan (Donatus, Ioannes) 406, 411, 412, 414, 415, 416

Dončević, Ivan 99

Dopfl, konzul 472

Doré, Gustav 46, 47

Doroghy, Zvonimir 27, 52

Dostojevski, Fjodor Mihailovič 24, 35, 79, 87, 96, 478

Dragićević, Rajna 192, 194, 200

Drašković, Gašpar 392

Dubajić, Dejan 468

Dubajić, Dušan 468

Dubajić, Stevo 468

Dujšin, Dubravko 464, 469

Dukat, Zdeslav $51,53,54$

Dukić, Davor 70, 87, 135, 145, 211, 222, 231, 235

Durkheim, Emile 426, 428, 437

Durman, Milan 444, 447

Dušan, Stefan Uroš IV., car 352

Dušanić, Dunja 304, 313

\section{Dř}

Džahiz (Al-ğāhiz) 294, 301

\section{$\oplus$}

Đorđević, Bojan 13, 141

Đurđević, Darija 261

Đurđević, Goran 16, 265, 278

Đurić, Miloš N. 49, 50, 51, 52, 53

\section{E}

Edmondson, Jonathan $375,377,383,396$, 397
Edward (Edvard) II., kralj 484

Edwards, Derek 124, 125

Edwards, Paul 182, 189

Einstein (Ajnšatajn), Albert 306

Eitelberg, Abraham 426, 446

Ellias, Norbert 489, 492

Epiktet 34

Epikur 15, 34, 179, 181, 184, 185-199, 189, 214, 223

Erdödi, Thomas, ban 414

Eshil 10, 23, 24, 31, 34, 35, 36, 48-51, 52, 53, 58

Estok, Simon C. 263, 278

Euripid 35

Even-Zohar, Itamar 120,125

\section{F}

Faberio, Matija, vicenotar 405

Faberius, Matija, senator 410

Faberius, Toma, gradski prisjednik 410

Fabrio, Nedjeljko 440, 441, 447

Fabris, Wendy B. 344, 349

Farlati, pjesnik 387

Farlatti, Daniele 387, 396

Farrell, Susan 261, 263, 271, 278, 280

Fā'ūr, 'Alī 301

Faur, Elena 195, 200

Faustina, Mlađa, carica 374

Faustina, Starija, carica 374

Febvre, Lucien 421-422, 447

Fejfer, Jane 366, 367, 368, 374, 377

Feldman, Fred 179, 181, 189, 327, 337

Ferrero, Guglielmo 27

Fijan, Andrija 478

Filipčić, Ivan 393, 396

Filipović, Ivo 474

Filo, Jože 92, 101

Fincher (Finčer), David 361

Finci, Eli (Финци, Ели) 194, 114

Fiocchi Vincenzo, Nicolai 383, 397

Fischer, John Martin 185, 189

Flaubert, Gustav (Flober, Gistav) 35, 169, 177

Flores Moreno, Cristina 195, 200

Flynn, Thomas R. 173, 177

Folena, Gianfranco 286, 292 
Foscolo, Ugo 16, 17, 34, 281-291, 292

Foucault, Michel 16, 241, 244, 245, 246

Frangeš, Ivo 490

Franičević, Marin 25, 32

Franjo Josip, car 441

Fraschetti, Augusto 372, 374, 377, 378

Freud, Sigmund (Frojd, Zigmund) 15, 17, $214,215,218,219,221,223,303,305-$ $306,310,312,313,314,429,430,435-$ 436, 439, 447

Freudenreich, Aleksandar 472-473

Froman, Margareta 466, 467

Fromm, Harold 262, 264, 278, 279

Frye (Fraj), Northrop 104

Fukec (Fukecz), Barbara 410

\section{G}

Gabričević, Branimir 384, 396

Gaedtke, Andrew 439, 447

Galimberti, Sandor 433

Garenne, Michel 458, 461

Gauthier, Nancy 382, 383, 384, 396

Gavella, Branko 466, 468

Gavrilović, Milomir 131, 145

Gay, Peter 432, 433, 436, 447

Geertz, Clifford 240, 247

Gerner, Elza $466,472,480$

Gerrard, Greg 268, 278

Gerstl, Richard 435

Gheerbrant, Alain 353, 363

Gibbs, Raymond 193

Gide (Žid), André 96

Giesey, Ralph 484, 492

Gijswijt-Hofstra, Marijka 439, 448

Ginsborg, Paul 485, 492

Gjenero-Margan, Ira 453, 461

Gladwin, Derek 263, 278

Gligo, Vedran 388, 391, 392, 396, 397

Globačnik, Matko 9, 17, 329, 332, 338, 381, 396

Glotfelty, Cheryll 16, 262, 264, 278, 279

Glunčić-Bužančić, Vinka 271, 277, 278, 279

Goette, Hans Rupprecht 367, 377

Goldstein, Ivo 402, 419, 464, 480, 497

Goldstein, Slavko 402, 419, 464, 480, 497
Gordon, Peter A. 329, 337

Gortan, Veljko 54, 390, 396

Gostić, Josip 478

Gotovac, Jakov 464, 472, 477

Gotthelf (Gothelf), Jeremias 136

Grabar, Mato 404, 419

Grabar, Oleg 372, 378

Gračan, Mihajlo 94

Grba, Darinka 468

Grba, Pajo 466, 468

Greenberg (Grinberg), Gary 27

Gregorović, Maja 195, 201

Gregorovius, Ferdinand 27

Grgić Maroević, Iva 16, 282, 285, 286, 292

Grgić, Kristina 271, 278

Grković, Mate 478

Grmek, Mirko Dražen 20, 451, 452-456, 461, 462

Grosz, George 422, 444

Grozaj, Vera 478

Grubačević, Mihajlo, sudac 406, 409, 413

Ğubaylī, Samī' Ğamīl al 296

Gumplowicz, Ludwig 435

Gundulić, Ivan 464, 477

Gvozden, Vladimir 169, 177

\section{$\mathrm{H}$}

Halbwachs, Maurice 437, 447

Hamann, Brigitte 428, 429, 447

Hamdani, Abu Firas al 293

Hamvaš, Bela 212, 223

Handley, Mark A. 383, 396

Hanibal, vojskovođa 29

Hanžek, Ljudevit 333, 338

Harambašić, August 441

Harari, Yuval Noah $16,261,265-268,270$,

$273,276,277,278,280$

Harmoš, Oskar 466

Harpman, Geoffrey 151, 156

Harris, Paul L. 316, 325, 326

Hartman, Lavoslav 28, 52

Hārūn, 'Abd as-Salām 294

Hatze, Josip 422

Healy, Roisin 422, 447

Hebel, Johann Peter 136, 142-143

Hegedušić, Krsto 475, 476 
Hegel, Georg Wilhem Fried rich 329

Heidegger, Martin $10,17,18,79,80,87$, $176,327-336,338-339$

Heinz, Josip 472

Hemingway, Ernest 271, 276, 278

Henckmann, Wolfhart 329, 338

Hercigonja, Eduard 331, 338

Herkov, Zlatko 492, 413, 419

Hertz, Robert 483, 492

Herzl, Theodor 430

Hesberg, Henner von 365, 366, 377, 378

Hesse, Hermann 422, 426

Heziod 34

Hitchcock, Alfred Joseph (Hičkok, Alfred) 353

Hobbes, Thomas 241, 247

Hollaman, Keith 344, 349

Holjevac, Željko 432, 447

Homer 10, 15, 23, 34, 44, 50, 56, 289, 290, 297, 301

Horacije 27, 32, 34, 40, 43, 84, 299

Horvat, Anđela 392, 396

Horvat, Josip 431, 447

Horvat, Joža 90, 229, 232, 283

Horvat, Matija (Horvath, Matthias) 410

Horvat, Saša 381, 396

Horvat Pintarić, Vera 442, 447

Horvath, odnosno Halambek (Horvath aliter Halambegh), gospođa 407, 408, 409, 411, 412, 416

Hosu, Stjepan 54

Howarth, William 264

Hribar, Branko 423

Hristić, Jovan $15,105,106,113,114,203-$ 209, 210

Hugo, Victor (Иго, Виктор) 79, 87

Humanić, Andrija (Humanich, Andreas) 410

Hume, David 332

Husić, Snježana 52

Hüsken, Wim 484, 493

Husserl (Huserl), Edmund 13, 118, 125, 329, 330, 334

Hutinecz, Georgius 408

Huxley, Aldous 16, 237

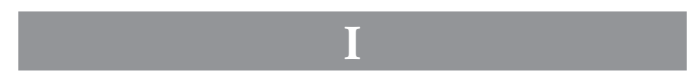

Ibsen, Henrik 478

Ilić, Anđelka 468, 472

Ilić, Darinka 468, 472

Ilić, Sima 468, 470

Ilić, Veljko 470, 472

Ilijašić, Jakov 410

Ilijić, Stjepko 285, 288

Inger, Iacob (Hynger, Jacobus) 406, 409, $412,413,414,415,416$

Ivan Ravenjanin, nadbiskup 384-385

Ivan, svećenik 384

Ivana iz Sirmija 384

Ivanić, Dušan (Иванић, Аушан) 73,87

Ivanišević, Igor 354, 360

Ivanišević, Milan 389, 396

Ivanišin, Nikola 491

Ivanković, Vedran 430, 447

Ivšić, Stjepan 331, 336

Izokrat 34

Izutsu, Toshihiko 295, 298, 301

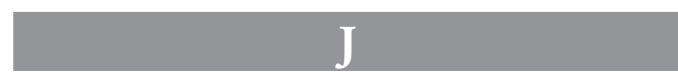

Jagatić, Doroteja (Jagatich, Dorothea) 407

Jakelić, C. 464

Jakšić Binički, Ruža 468

Jakšić, Nikola 384, 396

Jalland, Patricia 436, 447

Jambrehović (Jambrehovich), Gaspar, krznar $40,406,410,411,412$

Jambrišak, Tatjana 354, 357, 363

Janeković Römer, Zdenka 411, 419

Janik, Allan S. 440, 447

Jankélévitch (Jankelevič), Vladimir Viktor 176

Jarnević, Dragojla 353

Jaspers, Karl 10, 17, 18, 327, 328-331, 33336, 338-339

Jekić, Dragoljub (Јекнић, Арагофуб) 316, 326

Jelčić, Dubravko 45, 52, 53

Jelena, kraljica $385,386,389$

Jeličić, Živko $\quad 63,67$

Jelić-Butić, Fikreta 464, 465, 480

Jeremić, Dragan 135, 484, 490 
Jernej, Josip 285, 288, 292

Jeronim, sv. 383

Jiranek, Miloš 433

Johansson, Jens 327, 337

Johnson, Julie M. 447

Johnson, Mark 193, 194, 201

Johnson, Nail P. A. S. $\quad 454,461$

Johnston, Alexandra F. 484, 493

Johnston, William M. 439, 433, 434, 435, 440, 447

Joksimović, Žarko 113

Joshel, Sandra R. 373, 374, 375, 376, 377

Joszef, Attila 433

Jovanović, Asja 113

Jovanović, Slobodan 304, 314

Jović, Bojan 9, 10

Joyce, James 422

Juhasz, Gyula 433

Jukić, Vlado 441, 447

Jukić, Zlatan 23, 53

Jurčić, Vladimir 431, 432, 444, 447

Jurišić, Blaž 328, 333, 336

Jurišić, Šimun 38,53

Jurković, Miljenko 384, 385, 396

Justinijan, car 366

\section{K}

Ka'b, bin Zuhejr (Ka'b bin Zuhayr) 297

Kafka, Franz 161, 426

Kajs, Imrul (Qais, Imru’ al) 294

Kalanj, Rade 433, 447

Kaleb, Vjekoslav 91

Kalemer, Aurelije 55, 91, 99, 344

Kalinić, Antun Branko 367, 377

Kalpurnija, Cezarova žena 34

Kaminski, Martin 27, 53

Kampuš, Ivan 409, 419

Kant, Immanuel 18, 327-334, 335, 338

Kantorowicz, Ernst Hartwig 484, 485, 492

Kanjo, Zoltan 119, 125

Karahasan, Dževad 10, 18, 341-348, 349

Karaman, Ljubo 387,396

Karbić, Damir 381

Kardoš, Aleksandar 475

Kardoš, Olga 475

Karlo VI., car 484
Kaštelan, Jure 490

Katalinić Jeretov, Rikard 430

Katarina, kći Bele IV. 389

Katičić, Radoslav 385, 387, 389, 396

Katić, Lovre 385, 396

Katon, Marko Porcije Mlađi 27, 29

Katon, Marko Porcije Stariji 26, 27, 28, 29, 34

Katul, filozof 25

Katušić, Ivan 225, 226, 230-234, 235, 236

Kavić-Kardoš, Vojko 474-475

Kayser, Wolfgang 154

Kazimirović, Radovan N. 352, 363

Keglević, Šimun 393

Kelečić, David 354

Kermode, Frank 165, 167-168, 173, 175, 176, 177, 178

Kerrigan, Michael 22

Khaill, Ivan, gradski kapetan 406

Kierkegaard, Søren 329, 334

Kirša, Ingrid 269, 278

Kišs, Danilo 100, 131, 212

Klačić 37 v. Claccich

Klaić, Bratoljub 38, 44, 46, 52, 53, 54

Klaić, Nada 390, 396

Klaić, Nikola 38, 51

Klaić, Željko 53

Klee, Paul 422

Klikovac, Duška 200

Klimt, Gustav 434, 435

Knežević Ivašković, Milka 354, 355

Koch, Guntram 368, 369, 378

Kocijan, Gregor 93, 95, 101

Koharić, Janko 437, 438

Kohn, Gezo (Kon, Geco) 28, 130

Kolar, Slavko 89, 99, 101

Kolar-Dimitrijević, Mira 385, 397

Kombol, Mihovil 52, 285

Konstantinović, Radomir 15, 217, 223

Konjević Milošević, Nikolina $\quad$ 148, 156

Kopić, Mario 163, 164

Korać, Stanko 23, 24, 28, 32, 38, 49, 52, 53, 148, 156, 283, 291

Kordić, Ivan 330, 338

Korolija, Mirko 38, 226

Koron, Alenka 101

Kosmač, Ciril 99-100 
Kostić, Josip 33, 39, 53

Kovačić, Ante 441

Kovačić, Mirko 38, 53

Kovačić, Viktor 438

Kovič, Kajetan 95, 101

Kozak, Juš 94

Koziak, Mihael (Kozyak /Koziak, Kosziak/, Michael), gradski kapetan 405, 408

Kraljeva, B. 464

Kraljević, Miroslav 431, 442, 445

Kraljević, Vinko 113

Kramer, Samuel Noah 27

Kravar, Zoran $15,72,87,211,212,218$, 223, 232, 235, 446

Krčelić, Vatroslav 464

Krescencije, nadbiskup 385

Krivošić, Stjepan 402, 419

Krizman, T. 478

Krklec, Gustav 283

Krleža, Bela 466-467

Krleža, Miroslav 10, 13, 16, 17, 303-313, 314

Kroeber, Karl 262, 278

Kropš, I. 464

Krose, Hermann Anton 425, 446

Kršnjavi, Izidor 431, 437

Krušvar, Zoran 354, 361, 362

Kselman, Thomas A. 436, 447

Ksenofont, povjesničar 27

Kübler-Ross, Elisabeth (Kjubler Ros, Elizabet) 127

Kučera, Elza 333, 336

Kugli, Stjepan 28

Kuhar, Franjo 113

Kukuljević Sakcinski, Ivan 392, 393, 395, 396

Kulišić, Špiro 353, 363

Kuntić-Makvić, Bruna 10

Kušević (Kussevich), Helena 407, 408, 410, 413, 415, 416

Kutejba, Ibn (Qutayba, Ibn) 296, 297, 301

Kuzmić, Aleksandra 12

\section{L}

La Rochefoucauld, François de 169

Lacan, Jacques 163
Ladan, Tomislav 490

Lakoff, George 193, 194, 199, 201

Lalić, Mihail 99-100

Larkin, Margaret 300, 301

Lasch, Richard 426

Lasić, Stanko 305, 314

Lasko, Emil 329

Laszowski, Emilij 418

Laurenčić, J. 464

Lazar, Alice 316, 326

Lazarević Giacomo, Persida Di 169, 177

Le Blant, Edmond Frédéric 383, 396

Le Rider, Jacques 439, 447

Lebid bin Rabi’a (Labīd bin Rabīáa) 296, 301

Leburna, glumac 370-371, 372, 379

Lehar, kompozitor 474

Lehman (Leman), Hans Thies 104

Lehmann, Karl 330, 331, 338

Leibniz (Lajbnic), Gottfried Wilhelm 79

Leigh Littlechilds, Rebecca 383, 397

Lemon, Lee T. 119, 126

Lengel-Krizman, Narcisa 470, 480

Leopardi, Giacomo 34, 282

Leskovar, Janko 423

Levanat-Peričić, Miranda 261 27, 276, 278, 279

Levanić, Karmen 406, 419

Lewis, Charlton T. 39, 53

Liddell, Henry George 33, 39, 53

Lim, Richard 372,378

Lisac, Josip 331, 338

Livadić, Vjekoslav 441

Lompar, Miro 308, 312, 314

Loschmidt, Josef 440

Lotman, Jurij Mihajlovič 13, 148-150, 156

Lovrenović, Dubravko 381, 383, 397

Lovro, dr. 74

Lovro, nadbiskup 385, 388

Lozovina, Vinko 285, 288

Lucii, Ioannis 387,397

Lucio, Giovanni 37, 53

Luckmann, Thomas 118, 125

Lučić, Ivan 37

Lučić, Josip 385, 397

Lukan, Marko Anje, pjesnik 389

Lukrecije, Tit, filozof 25, 181, 189, 285 
Luper, Steven 179,89

Lužina, Jelena 468, 480

Lynn Ryan, Katherine 436, 448

Lynn Sebesta, Judith 367, 378

\section{LJ}

Ljeskov, Nikolaj Semjonovič 136

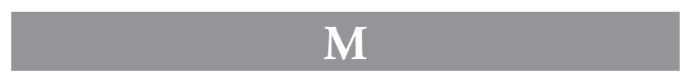

Macan, Darko 354, 361

Macho, Thomas 22

Mađarević, Sven 113

Magjer, Drago 443

Mahler, Alma 434, 440

Mahler, Gustav 435, 440

Mahon, Margaret M. 315, 316, 326

Maiksner, Franjo 33, 39, 53

Majakovski, Vladimir Vladimirovič 422

Majić, Ivan 14

Makanec, Julije 334, 336

Manderson, Desmond 434, 447

Mandrou, Robert 422, 447

Manet, Edouard 422, 423

Mann, Thomas, (Man, Tomas) 13, 94, 426

Manojlović, Gavro 27

Marafioti, Nicole 485, 492

Maras, Mate 52

Marčetić, Marija 22

Maretić, Tomo 297, 301

Margareta, kći Bele IV. 389

Margolin, Uri 119, 126

Maričić, J. 464

Marija Terezija, carica 288

Marin, Emilio 382, 383, 384, 396, 397

Marin-Arrese, Juane I. 195, 196, 199, 201

Marinetti, Filippo Tommaso 422

Marinković, Dušan 23, 24, 25, 26, 27, 51, $52,53,54,83,87,107,251,259,283$, 286, 291, 291

Marinković, Konstantin 353

Marinković, Ranko 91

Marjanić, Suzana 261, 262, 279

Marjanovć, Branko 489

Marko Aurelije 368, 374
Marković, Bojan (Марковић, Бојан) 316, $317,321,326$

Marković, Franjo 333

Marković, Miroslav 385, 387, 388, 389, 397

Markovski, A. 478

Maroević, Tonko 31, 52, 53, 181, 189, 250, 259

Marohnić, Jelena 41, 44, 53

Maron, Vergilije Publije v. Vergilije

Marquez, Gabriel G. 344

Martin, Mojmir 465, 480

Martin, nadbiskup 385, 386, 388, 389

Martinac Kralj, Lada 113

Martinović, Milica 214, 223, 306, 313

Martinović, Žarko $214,223,306,313$

Marucchi, Orazio 383, 397

Marušić, Ivan (Marussich, Joannes) 403

Masaryk, Tomaš Garrigue 426, 446

Masereel, Frans 436

Mašanović, Dimitrije 489

Matačić, Lovro 478

Matavulj, Simo 91, 491

Maté, Miha 90, 101

Matijašić, Robert 382, 386, 397

Matijević Sokol, Mirjana 19, 383, 384, 385, 386, 387, 391, 392, 393, 395, 397

Matković, Hrvoje 463, 464, 465, 480

Matković, Marijan 89, 91, 101, 442, 446

Matković, Miloslav 104, 114

Matković, Stjepan 431, 442, 447

Matoš, Antun Gustav 183, 423, 430-431, 434, 441-443, 446

Maurek, Katarina (Catharina) 403

Mavrin, Igor 354, 357

Mazzoleni, Danilo 383, 397

Mažuranić, Fran Vladimir 430

Mažuranić, Ivan, ban 441

McCarthy, Cormac 274, 275

McCormick, John P. 329, 337

McHale, Brian (Mekhejl, Brajan) 118, 120, 121,126

McLean, Bradley H. 382, 397

Meco, Zenaida 195, 201

Medick, Hans 433, 446

Melanija, ropkinja Cezernija Avita 373, 374

Meničanin, Pjer 113

Mesterhazy Pongracz, Klara 434 
Mesterhazy, Kalman 433, 434

Mey, Jakob 199, 201

Meyer-Fraatz, Andrea 277, 279

Migotti, Branka 18, 368, 373, 374, 375, 376, 378

Mihailović, Barbara 345

Mihajlović, Jevstatije 353

Mihajlović, Mića 321

Mihalić, Slavko 490

Miklić, Josip 54

Milarić, Ivica 354, 356

Miles, Richard 372, 378

Milošević, Nikola 207, 210

Milovanović, Milovan 443

Milović, J. M. 33

Minois, George (Minoa, Žorž) 422, 428, 447

Miossich-Cacich, Paolo Clemente 37

Mirković, Leo 472

Mirković, Miloslav 104

Mišić, Boris 354, 359, 360

Mitrović, Marija 12

Mlakić, Josip 16, 261, 263, 271-275, 276, 279, 280

Montaigne, Michel de (Montenj, Mišel) 15, 34, 94, 177, 203, 205-206, 210, 215, 223

Morgenbesser, Sidney 182, 189

Morin (Moren), Edgar 165, 169, 177, 178

Morley, John 436, 447

Morović, Hrvoje 388, 397

Morselli, Enrico 426

Morton, Timothy 275, 279

Mosinger Popović, Mila 474

Mosinger, Franjo 474

Mouritsen, Henrik 373, 374, 376, 378

Mozart, Wolfgang Amadeus 478

Mueller, Juergen 454, 461

Muhamed, prorok 297

Munch, Edvard 436

Musil, Robert 426

Musulin, Bogdan 429

Mutanabbi (Al-Mutanabbī) 293, 299, 300, 301

\section{N}

Nagel, Thomas 179, 189

Nancy, Jean-Luc 174, 177
Napoleon, Bonaparte 16, 283, 284, 287

Natorp, Paul 329

Naumova-Orlova, Olga 466

Nazor, Ante 463, 466, 480

Nazor, Vladimir 281, 285, 288, 292

Nedić, Marko 304, 314

Nelson, Leonard 329

Nemec, Krešimir 13, 39, 52, 53, 83, 87, 150, 151, 152, 156, 347, 247, 250, 252, 259, 264, 279

Nepot, Kornelije 27, 29, 30, 54

Neralić, T. 478

Nevija Romanija 375-376

Nietzsche, Friedrich 168-169, 178, 329, 334

Nikefor II. Foka, car 293

Nodier (Nodije), Jean Charles Emmanuel 136

Nodilo, Natko 438

Nogić, Srđan 113

Novak, A., kapelan 468

Novak, Josef 425, 448

Novak, Viktor 385, 387, 397

Novaković, Darko 51

Novaković, Marija 441

Novaković, Snježana 354

Noymer, Andrew 458, 461

Nubata as-Sa' di, Ibn 293

Nučić, H. 464

Nüll, Eduard van der 435

Nyiri, Christoph J. 425, 427, 434, 448

\section{$\mathrm{Ni}$}

Njegoš, Petar Petrović 86

\section{O}

Ognjanović, Dejan 352, 353-354, 363

Olstein, Diego 276, 279

Oltvanji, Oto 353, 354, 363

Olujić-Lešić, Grozdana 34, 54

Onfray, Michel (Onfre, Mišel) 168, 173, 178

Opačić, Zorana (Опачић, Зорана) 316, 326

Opašić, Maja 195, 201

Oppermann, Serpil 275, 279 
Orljak, Petar 111

Orobović, Joco 466

Ortall, Jacopo $367,368,378$

Orwell, George 16, 238, 359

Osimo, Bruno 282, 286, 290, 292

Öttingen, Alexander von 426

Ovidije, Publije Naso 27, 40, 389

\section{P}

Pagliai, Francesco 286, 292

Palahniuk, Chuck 361

Palavestra, Predrag 351, 363

Panayotatkis, Costas 372, 378

Pančić, Josif 484, 493

Pantelić, Nikola 353, 363

Pantić, Mihajlo 152, 156, 307, 314

Parkinson Zamora, Louis 344, 349

Paronić, Samanta 22

Pascin, Jules 443

Pašić, Nikola 132

Pavelić, Ante $463,475,477,478,481$

Pavić, Željko 328, 331, 335, 338

Pavis, Patrice 194

Pavissich, Luigi Cesare 37

Pavletić, Vlatko 23, 25, 31, 52, 53, 54, 72, 87, 91, 490

Pavlina, Želimir 333,338

Pavlović, Cvijeta 277, 279

Pejčić, Grozda 22

Peleš, Gajo 79, 87, 231

Peričić, Helena 83, 87, 104, 114

Perić, Olga 385, 395

Perišić, Marija 265, 279

Perković, Renata 52

Petar Crni, mecena 385, 387, 388

Petar, nadbiskup 391

Petar, prior 384

Peterfy, Jenő 433, 435

Petković, Nikica 18, 343, 349

Petračić, Franjo 33, 39, 52, 53

Petranović, Teodor (Петрановић, Теодор) 145

Petras, Ana (Petrasz, Anna) 403

Petras, Ivan (Petrasz, Joannes) 403, 407

Petricioli, Ivo 384, 398
Petričević, Katarina (Petrichevich,

Catharina) 407, 408, 412, 413, 415, 416

Petrić, Hrvoje 402, 419

Petronije Arbiter (Arbitar) 34, 367, 377

Petrović, Petar Ž. 353, 363

Phillips Lovecraft, Howard (Filips Lavkaft, Hauard) 352, 363

Pičman, Josip 421, 438-439, 449

Pilar, Ivo 431, 432, 442

Pindemonte, Ippolit 16, 34, 393

Pistorius, Hermann Andreas 329

Platon 27, 31, 34, 301, 306, 334, 342

Plaut, Tit 34

Plinije, Gaj Cecilij Mlađi 39

Plinije, Gaj Cecilij Stariji 34

Plotin 34

Plutarh 23, 26, 27, 29, 30, 31, 39, 54

Podgorska, Vika 464

Pogan, Parsifal 354, 358, 359

Pöggeler, Karl 330, 338

Poklečki Stošić, Jasminka 381, 397

Polić Kamov, Janko 423, 441, 442

Polić, Branko 465, 480

Polić, Dragimir 442

Pompej, Gnej, vojskovođa 27

Popov, Raša (Попов, Раша) 316, 326

Popović, Edo 16, 261, 263, 171-275, 277, 279, 280

Popović, Radovan (Поповић, Радован) 316, 326

Porter, Peter 165,178

Porter, Roy 439, 448

Pošman, Gerda 104

Potokar, Tone 12, 90-93, 95, 96, 101, 102

Poturić, Grga 408, 410

Pozuinszky, Martinus 408

Pozvinski, Juraj, podsudac 408

Požar, Petar 464, 480

Požgaj, Judita (Posgai, Iuditta) 410

Pražić, Milan 316, 326

Prelog, Milan 470, 480

Premerl, Tomislav 438i, 448

Prévot, Françoise 382, 383, 384, 396

Prica, Čedo 91

Price, Henry Habberley 342, 343, 348, 349

Primorac, Strahimir 272, 279

Prister, Lada 392, 398 
Protrka Štimec, Marina $211,212,213,216$, 223

Proust, Marcel 422

Pucak, Mihal (Puszak, Michal), sudac 408

Purcell, Nicholas 366, 367, 378

Purthin, Ana 393

\section{$\mathbf{R}$}

Rabar, Ivan 27

Rabelais, François 150, 151, 154

Rac, Koloman 49, 52, 53

Racko, Ljerka 438, 448

Račić, Josip 421, 430-431, 449

Radev, M. 478

Radica, Bogdan 431, 448

Radmanović, Petar (Pero) 466, 468

Radulović, Jovan (Радуловић, Јован) 73, 87

Ragon, Michel 488, 492

Rakić, Vikentije 353

Rako, Biserka 328, 336

Rapanić, Željko 384, 395, 386, 387, 396, 398

Rapo, Dušan 148, 156

Raspor, Tizian 265, 279

Rašeta, Irena 354, 362

Raškaj, Slava 441

Ratkaj, Petar II. 392, 394

Ratkaj, Sigismund 393, 394

Raukar, Tomislav 384, 398

Ravija, Hammad ar 294

Ređep, Draško 315, 319, 324, 326

Reis, Marion J. 119, 126

Remarque, Erich Maria (Remark, Erih Marija) 13, 117

Rendić-Miočević, Ante 369, 378

Rendić-Miočević, Duje 382, 384, 385, 398

Repak, Salko 468

Reynolds, Paul Anthony 383, 398

Ricci, Giovanni 484, 493

Rickert, Heinrich 329, 330

Ricœur (Ricoeur), Paul 120, 126

Ringel, Erwin 429

Rismondo, Vladimir 23, 24, 54, 211, 223, 230, 235

Roazen, Paul 430, 448

Robnik, Saša 354, 359
Roić, Sanja 16, 169, 177, 281, 282, 292

Rojc, Nasta 431, 444-445

Roje, Ana 466

Roksandić, Drago 9, 70, 72, 73, 78, 83, 87, 104, 114, 130, 135, 145, 148. 152, 156, $211,222,223,230,231,232,235,468$, 480

Rollin, Charles 27, 28

Roman II., car 293

Romulijan, Kletije 375

Rorauer, Julij 421, 440, 449

Rosenbaum, Stephen E. 184, 189

Rosenberg, Jay F. 179, 180, 182, 189

Rosinić, Margareta (Rossinich, Margaretha) 410

Rotar, Janez 96, 97-100, 101, 102

Roth (Rot), Klaus 228, 231, 235

Roth, Joseph 426

Rousseau, George 440, 448

Rozanov, Vasilij 422

Rubachevich, Mihajlo (Rubacheuich, Mihaly) 406

Rudolf, kraljević 428

Rueckert, William 262, 279

Rugg, Julie 487, 493

Rukavina, Dario 354

Rulfo, Juan 10, 18, 341-348, 349

Rus, Josip 431, 448

Rutić, Joža 475, 480

Ryan, Marie-Laure 119, 125, 126

Ryznar, Anera 194

\section{S}

Saar, Ferdinand von 426, 435

Sabljak, Tomislav 148, 156

Sabotič, Ines 437, 448

Safo (Sapfo), pjesnikinja 25

Salisbury, Laura 440, 448

Salopek, Damir 38, 54

Sarajlić, Alma 345, 346, 349

Sarrazac (Sarazak), Jean-Pierre 104

Sartre, Jean-Paul (Sartr, Žan-Pol) 13, 103$114,115,177$

Sass, Louis A. 439-440, 448

Sasso, Antun 285, 288

Scheler, Max 329, 330, 334, 336 
Schiele, Egon 422, 439

Schlechta, Karl 168, 178

Schleifer, Michael 316, 325, 326

Schlosser, Joseph Calasanz (Josip Kalasancije) 441

Schnitzler, Arthur 426, 435

Schopenhauer, Arthur 329

Scott, Robert 33, 39, 53

Scotti, Mario 286, 292

Seemann, Otto 36-37, 54

Segedinac, Goran 354, 356

Seitz, Aleksandar 480

Sejfud-Davla, emir 293, 297, 300

Selenić, Slobodan (Селенић, Слободан) 104, 114

Selimović, Meša 14, 98, 159-164

Semino, Elena 192, 193, 201

Seneka 389

Senjanović, Petar 438

Serao, Matilda 422

Shail, Andrew 440, 448

Shakespeare, William 272

Short, Charles 39, 53

Sichtermann, Hellmut 368, 378

Sikirić, Zvjezdana 19, 412, 419

Silone, Ignazio 282

Simić, Novak 29

Simonid s Keja, pjesnik 382

Sindičić Sabljo, Mirna 213, 223

Sironić, Milivoj 51

Skok, Petar 385, 387, 395, 397

Skrigin, Žorž (Georg) 466

Sladić, Rade 470, 472, 473

Slaviček, Antonin 433

Slaviček, Milivoj 490

Slavnić, Radoslav 354, 358-359

Smrekar, Milan 427, 441, 446, 453, 461

Snillar, Ivan (Snellar, Snelyar, Snyelyar, Snyellar, Sniellar, Sniellyar, Zniller, Ioannes), gradski prisjednik 406, 407, 409, 414, 416-417

Sofoklo 19, 23, 24, 34, 35, 50,

Soher, Dubravko 354

Sokol, Vladimir 385,397

Sokrat 27, 250

Solar, Milivoj 192, 201

Somlo, Felix 433
Specht, Richard 440

Speece, Mark 316

Spencer Northcote, James 381,398

Squire, Louise 261, 263, 274, 275, 279, 280

Srdarević-Prkut, Mile 29, 139, 140, 150

Srkulj, Stjepan 27

Stahl, Ernst Leopold 437, 448

Stamać, Ante 196, 198, 201

Stamenković, Vladimir 104, 114

Stanković, Borislav 69

Stanojević, Mateusz-Milan 194, 195, 201

Starčević, Ante 477-478

Starčić, Viktor 111

Steen, Gerard 192, 193, 201

Stepanić, Ivan, sudac 404, 414

Stepinac, Alojzije, nadbiskup 477

Stieg, Michael 153, 156

Stifter, Adalbert 435

Stipetić, Vladimir 335, 338

Stivelman, Barnet P. 456, 461

Stojanović, Dragan (Стојановић, Араган) 82,87

Stojković, Pavao 466

Stošić, Ivan 472

Strabo(n), povjesničar 43

Strange, Julie-Marie 436, 448

Stražnicki, Stanislav 472, 474

Strecha, Mario 438, 448

Strozzi, Tito 464, 466, 470, 472, 475, 477

Stuparić, Darko 475, 480

Stuparić, Nikola (Sztuparich, Nicolaus), župnik 407, 411, 412

Suić, Mate 385, 398

Sumi, Geoffrey 372, 378

Sumić, Stana 466

Suppes, Patrick

Svačić, Petar 477

Svetlični, Vladimir 466

Svetonije, Gaj Trankvil, biograf 30, 39, 54

Svilović, Luka 285, 288

Szikszay, Ferenc 433

\section{ড̌}

Šaban, Damir 113,354

Šakić Ristić, Iva 13

Šākir, AḤmad MuḤammad 297, 301 
Šanqịțī , AḤmad al-Amīn aš 294, 295, 296, 301

Šantić, Stjepan 475

Šarčević, Stevan 354, 356

Šašel Kos, Marjeta 370, 378

Šeatović, Svetlana 15, 169, 177

Šega, Milan 92, 101

Šegedin, Petar 90

Šegvić, Neven 438, 448

Šen, Edo 438

Šercer, Marija 416, 419

Šešelj, Zlatko 50, 54

Šicel, Miroslav 488

Šimanović, Ana (Simanovich, Anna) 407, 409, 410

Šimenc, Stanko 92, 101

Šimetin Šegvić, Filip 19, 434, 448

Šimetin Šegvić, Filip 19

Šimleša, Dražen 277, 279

Šimunić, August 468

Šišić, Ferdo 386, 398

Šitum, Tea 273, 279

Škiljan, Filip 468, 480

Škreb, Zdenko 130, 145

Šnajder, Đuro 34, 89, 90

Šorak, Dejan 113

Šram, Ljerka 434

Štajner, Karolina 468

Štampar, Andrija 460

Štampar, Emil 12, 83, 94, 101

Štimac, Josip 398

Štrk, Ivan 470, 471

Štuka, Tvrtko 354

Štulac, Ana (Stulacz, Anna) 403

Štursa, Jan 433

Šubić, Mladen III. 390

Šukić, Andrija 466, 468

Šuklje, Rapa 92, 101

Šunjić, Ankica 38, 53

Šušić, Ilija 466, 468

Šutalo, Goranka 135, 145

Švelec, Franjo 490

\section{$\mathrm{T}$}

Taaffe, Eduard von 428

Tacit, Publije Kornelije 30, 39
Tadić, Ivan 331, 338

Tadić, Ljubomir 176, 178

Tadić, Vinko 261

Tadijanović, Dragutin 430, 448

Tahi, Franjo 392

Tajović, Lado 100

Talanga, Josip 51

Talwar, Victoria 316, 325, 326

Tanhofer, Tomislav 85, 104, 111

Tarafa, bin Al-Abd 294, 295

Tesla, Nikola 484, 493

Tešić, Gojko 304, 308, 312, 313, 314

Tešić, Iva 17

Thausing, Moriz 435

Tišma, Aleksandar 139, 140

Tito Livije 27

Todorov, Cvetan 227, 235

Tolstoj, Lav Nikolajevič 48

Toma Akvinski 343, 348

Toma Arhiđakon 385, 391, 395

Tomanović, Lazar 285, 288

Tomasevich, Jozo 464, 480

Tomašić, Nikola, ban 440

Tomić, Petar 27

Torney-Purta, Judith 316, 326

Toynbee, Jocelyn M. C. $366,367,378$

Traina, Giusto 372,378

Trakl, Georg 434, 435

Trček, Tjaša 264, 279

Trebješanin, Žarko 305, 313

Trepše, Marijan 464

Tribinacz, Nikola, vicenotar 406

Trnina, Milka 478

Trnski, Ivan 285, 288

Trotter, David 440, 448

Trputec-Teree, Irma 437

Tugendhat, Ernst 15, 217, 221-222, 223

Turcan, Robert 368, 378

Turner, Mark 193, 201

Tvrdakov, Ivan, nadbiskup 384, 385

Tynan, Kenneth (Tajnan, Kenet) 106

\section{$\mathrm{U}$}

Ujević, Tin 52, 423, 443-444, 448

Umejja bin Abi Salt (Umayya bin abī aṣȘalt) 296, 301 
Uranić, Igor 382, 398

Urem, Mladen 442, 448

Uzolin, Ivan (Uzulin, Joannes) 403, 404

\section{V}

Vaglio, Luca 73, 87, 211, 223

Vaić, Đorđe 466, 468

Valent, rob Cezernija Avita 373-374

Valentina, ropkinja Cezernija Avita 374

Valentić, Mirko 392, 398

Vallin, Jacques 452, 456, 461

Valjavec, Mato 39, 52

Vasić, Dragiša 10, 17, 303-313, 314

Vaupotić, Miroslav 312, 314

Vavra, N. 464

Veigl, Hans 440, 447

Vekenega, opatica 385, 388-390, 395

Veledinović, Stevan (Stjepan) 468

Venturi, Lionello 282

Verbič, Benjamin 96

Vergilije, Publije Maron 10, 23, 27, 34, 40, 44, 45, 46, 47, 54, 393, 389

Vernić, Juraj 405, 416

Vernić, Mihajlo, senator 407

Vernić, Zdenko 331, 333, 336

Verzár Bass, Monika 367,378

Veselinović (Ueszellinouich), Helena 407, 408, 411-413, 415-418

Vešović, Radonja 99

Vežić, Vladislav 285, 288

Vidaković, Milovan 353

Vidal, Michel 367,378

Vidrić, Vladimir 441

Vilar, Jean (Vilar, Žan) 106

Vilim, princ 389

Vinci (Vinči), Leonardo da 177

Vismara, Cinzia 367, 368, 378

Vital, nadbiskup 385

Vitez, Grigor 89, 101

Vittorio Emanuele I., kralj 485

Vladanović, Matko 274, 279

Vladušić, Slobodan 206, 210

Vodopivec, Nikola 475

Vojnović, Ivo 44

Vojnović, Martin 414
Vojnović, Matija (Voinovich /Voynouych/, Matthias), kovač 406, 407, 409, 412

Vovelle, Michel 21-22, 422, 448

Vratović, Vladimir 390, 396

Vrban, Darko 353, 354, 363

Vučetić, Radin 96, 101

Vučković, Radovan 304, 305, 313, 314

Vujasinović, Jovo 491

Vujatović, Stevan (Stjepan) 466, 468

Vujnović, Milan 466, 470

Vuković, Stjepan 468

\section{W}

Wagner, Adolf 426

Walter, Tony 484, 492

Weaver, John C. 422, 426-427, 448

Weber, Max 437

Weininger, Otto 434

Weiss, Nathan 436

Westcott, William Wynn 425, 448

Wheeler, Michael 436, 448

White, Hyden 344-345

White, Morton 182, 189

Wiener, Philip P. 263, 278

Windelband, Wilhelm 329

Wisser, Richard 330, 338

Wittgenstein, Ludwig 435, 440

Wolff, Larry 429, 448

Woodthorpe, Kate 487, 493

Wynn Westcott, William 425, 448

\section{Y}

Yeats, William Butler 272

Yobs, Steven 272

Young, David 344, 349

\section{$\mathrm{Z}$}

Zagorac, Milan 442, 448

Zajc, Ivan 478

Zalar, Ivo 52

Zanker, Paul 365, 377, 378

Zeligman, Kurt 352, 363

Zidić, Igor 44, 52, 431, 448 
Zimmermann, Stjepan $10,17,18,327-336$, 337, 338-339

Zrinski, Nikola Sigetski 392

Zuhejr, bin Abi Sulma 296, 299

Zupančić, Joža 90

Zvonimir, kralj 390-391

Zweig, Stefan 426

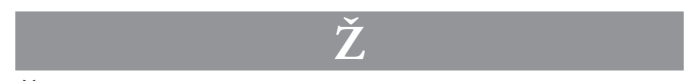

Žanko, Dušan 463, 464, 465, 480, 481

Žepić, Milan 39, 54

Žepić, Sebastijan 39, 52

Živković, Dragiša 59, 67

Žmegač, Viktor 152, 156, 436, 446 